\title{
Major lung resections with spontaneous ventilation: a narrative review
}

\author{
Cristina Diotti^, Davide Tosi
}

Thoracic Surgery and Lung Transplantation Unit, Fondazione IRCCS Ca' Granda Ospedale Maggiore Policlinico, University of Milan, Milan, Italy Contributions: (I) Conception and design: Both authors; (II) Administrative support: Both authors; (III) Provision of study materials or patients: Both authors; (IV) Collection and assembly of data: Both authors; (V) Data analysis and interpretation: Both authors; (VI) Manuscript writing: Both authors; (VII) Final approval of manuscript: Both authors.

Correspondence to: Cristina Diotti. Thoracic Surgery and Lung Transplant Unit, Fondazione IRCCS Ca' Granda Ospedale Maggiore Policlinico, University of Milan, Via Francesco Sforza 35, 20122 Milan, Italy. Email: cristina.diotti@unimi.it.

\begin{abstract}
In the last decades, technical improvements and the advance of innovative technologies have made thoracic surgery increasingly less invasive: video-assisted thoracic surgery (VATS) is now considered the best approach for early-stage non-small cell lung cancer (NSCLC) surgical treatment, and has become the first choice for many non-oncological thoracic diseases requiring surgery, with proved advantages in terms of less post-operative pain, fewer complications and a faster recovery. Combining minimally invasive surgical and anaesthetic techniques is one of the new frontiers of thoracic surgery: in this scenario, nonintubated thoracic surgery (NITS) is a promising approach to minimize risks related to general anaesthesia and intubation, promote a faster recovery and reduce hospital stay. Since its first reported applications for minor thoracic procedures in the late 90 s, NITS has emerged as a promising technique and it is recently being applied also to technical demanding major thoracic anatomical resections, such as lobectomies and segmentectomies, with encouraging results concerning feasibility, perioperative outcomes and complication rates. The oncological value of NITS is also being explored: recent studies show better compliance with adjuvant chemotherapy protocols and fewer events of toxicity in patients treated with NITS procedures. The aim of this narrative review is to investigate the role of NITS applied to major lung resections in terms of feasibility, short-term outcomes and applications in everyday clinical practice.
\end{abstract}

Keywords: Lobectomy; segmentectomy; major lung resections; non-intubated thoracic surgery (NITS); lung cancer

Received: 17 August 2021; Accepted: 27 September 2021; Published: 30 June 2022.

doi: 10.21037/asj-21-79

View this article at: https://dx.doi.org/10.21037/asj-21-79

\section{Introduction}

In recent years, remarkable progress in minimally invasive surgery and the spread of innovative technologies have led to significant improvements of video-assisted thoracic surgery (VATS), with proved benefits in terms of faster recovery, fewer post-operative complications and better outcomes if compared with traditional surgical approach (1). Historically, during VATS procedures intubated double- lumen anaesthesia was considered mandatory to obtain an optimal surgical field and to manipulate the lung, especially performing anatomical resections requiring a careful hilar and vascular dissection.

Pushing the limits of minimally invasive surgery also means reducing the invasiveness of anaesthetic techniques: in this perspective, non-intubated thoracic surgery (NITS) allows performing thoracoscopic procedures decreasing intubation-related risks and complications with similar

\footnotetext{
$\wedge$ ORCID: 0000-0001-8619-961X.
} 
surgical results if compared with intubated anaesthesia.

Technical demanding NITS thoracoscopic procedures, such as uniportal lobectomies and segmentectomies, are described to be feasible and safe: however, an adequate surgical learning curve, beginning from minor procedures such as pleural biopsies, pleurodesis and pulmonary biopsies is pivotal to approach challenging major anatomical lung resections. In addition, an efficient cooperation between surgical and anaesthesiological teams is essential to manage potential complications requiring immediate intervention.

The aim of this review is to explore the role of NITS applied to major lung resections in terms of feasibility, safety and post-operative outcomes. We present the following article in accordance to the Narrative Review reporting checklist (available at https://asj.amegroups.com/ article/view/10.21037/asj-21-79/rc).

\section{Methods}

A literature search was conducted by the authors to identify all published articles on NITS and major lung resections. PubMed and Embase were consulted matching the terms "Non intubated", "major lung resections", "lobectomy", "segmentectomy" "thoracoscopy" "video-assisted thoracic surgery" with "AND" until 30th April 2021. The search was extended by consulting the listed references of each article. Abstracts and non-English-written articles were excluded from the review.

\section{Technical aspects}

Traditionally, general anaesthesia with tracheal intubation and double lumen tube was considered mandatory to perform pulmonary resections, allowing single lung ventilation and ensuring an adequate surgical field during the manipulation of the lung. With the advent of minimally invasive thoracic surgery, appropriate single lung ventilation played a key role in major lung resections to optimize the exposition of vascular structures in a thoracoscopic view, allowing a safe hilar dissection.

However, since the early 2000s, Pompeo et al. described the first successful thoracic surgery procedures-mainly non-anatomical lung resections-performed without intubation, using epidural anaesthesia (2). Since then, NITS was applied with surprising results also to technically more challenging procedures, such as major anatomical resections. The first reported non-intubated VATS (NiVATS) major resections are from 2007 (3), and the first successful uniportal NiVATS lobectomy was performed by Gonzales-Rivas in 2014 (4).

While NITS minor procedures can be performed safely also at the beginning of the learning curve as first non-intubated experiences, NITS major resections are technically demanding procedures requiring an experienced surgical team and should be performed by highly trained surgeons in high volume NITS centers.

The main advantages of NiVATS procedures are the reduced rates of post-operative adverse effects of general anaesthesia (vomiting, headache...), often requiring more medications. Intubation-related airway lesions, tracheal traumas, ventilator-induced lung injuries and sore throat are minimized without a large-calibre double-lumen tube. Furthermore, the risk of bronchospasm in predisposed patients-chronic obstructive pulmonary disease (COPD) or asthmatic subjects-is reduced due to decreased airway irritation. Moreover, the absence of muscle relaxants avoids the residual neuromuscular blockade, a relevant risk factor for the development of early post-operative respiratory complications (hypoxemia, impaired ventilation, atelectasis and pneumonia) (5). Considering elderly patients, neurological complications such as delirium and cognitive dysfunction after intubation are common: avoiding general anaesthesia reduces the risk of developing persisting postoperative neurological deficits (6).

\section{Patients' selection}

A careful selection of patients undergoing NITS anatomical lung resections is essential to obtain an optimal surgical result. Many studies dealing with anatomical lung resections and NITS recommend avoiding patients with body mass index $(\mathrm{BMI})>25 \mathrm{~kg} / \mathrm{m}^{2}$ : obeses' respiratory pattern is associated with deeper diaphragmatic and mediastinal movements, which can make difficult the dissection, in particular in proximity to vascular structures. Nonintubated thoracoscopic surgery is suitable in small-sized females (7), distinguished by a more favorable breathing pattern. Moreover, the small tracheal caliber of these subjects makes them more prone to potential tracheal damages during intubation.

Other relative contraindications to NITS are coagulation disorders, difficult airways, previous pulmonary resections or radiation therapy, cardiac and neurological disorders that could make more complex the surgical procedure or airway management.

NITS can be proposed also to elderly patients: NITS 
lobectomy can be considered as a valid alternative to intubated VATS in selected geriatric patients ( $>65 \mathrm{y} / \mathrm{o})$, with results comparable to conventional procedures in terms of complications rates and surgical outcomes (6).

\section{Anaesthesia}

From an anaesthesiological point of view, NITS procedures are performed without inhalation agents and muscle relaxants; deep sedation is obtained using propofol and remifentanil infusion (8), or propofol alone with an effective intercostal block, reserving remifentanil only for breath rate control. A premedication of midazolam (0.15$0.25 \mathrm{mg} / \mathrm{kg})$ and atropine $(0.01 \mathrm{mg} / \mathrm{kg})$ and a nebulization of $5 \mathrm{~mL}$ of lidocaine 2\% 15-30 minutes before anaesthesia are useful to obtain optimal sedation, reduce secretions and avoid intense coughing during hilar manipulation. Starke et al. recently proposed a scheme of continuous infusion of dexmedetomidine during the surgery: the main advantages using dexmedetomidine are the prolongation of local anaesthetics effects and a lower risk of airway collapse if compared with other classes of sedatives (9).

Bispectral index sensor (BIS) is commonly used to monitor the depth of sedation, and standard monitoring of vital parameters includes non-invasive arterial blood pressure, pulse oximetry, electrocardiogram and heart rate, respiratory rate and end-tidal carbon dioxide measurement (10).

\section{Locoregional anaesthesia}

During NITS, locoregional anaesthesia together with effective deep sedation are pivotal to reduce movements and to achieve a surgical field as similar as possible to a conventional intubated thoracic surgery: either epidural, paravertebral or intercostal block can be used to obtain effective analgesia. Epidural anaesthesia is obtained using an epidural catheter inserted preoperatively into the T5/T6 thoracic interspace to achieve and maintain a sensory block between the T2 and T9 dermatomes using 2\% lidocaine. Paravertebral blockade is performed preoperatively with ultrasound-guided infiltration of ropivacaine $1 \%$ in close proximity to the vertebral transverse processes in four intercostal spaces (5 mL/space) (11). Intercostal block is performed intraoperatively injecting $0.5 \%$ bupivacaine in the intercostal space under direct thoracoscopic vision (generally from the third to the eighth intercostal nerves, $1.5 \mathrm{~mL} /$ space) (12). Locoregional anaesthesia is associated with better hemodynamic stability and postoperative respiratory function, a more effective postoperative analgesia, decreased surgical stress response and immunosuppression $(10,11)$.

Vagal nerve blockade, essential to minimize cough reflex during lung and airway manipulation, is obtained by injecting local anaesthetics (usually bupivacaine $0.25 \%$ ) in the paratracheal space on the right side and at the level of the aortopulmonary window on the left side.

\section{Ventilation}

During conventional intubated thoracic surgery, the exclusion from the ventilation of the non-dependent lung (obtained using a selective ventilation via a double-lumen tube) causes a ventilation/perfusion mismatch that can lead to hypoxemia and right-to-left intrapulmonary shunt. Furthermore, the use of muscle relaxants reduces the contraction of the dependent hemidiaphragm, worsening the ventilation of the dependent lung and increasing the risk of atelectasis even with a positive pressure mechanical ventilation.

During non-intubated surgery, lung deflation is obtained inducing a iatrogenic pneumothorax through thoracoscopic incisions under spontaneous breathing; the resulting lung collapse is more physiological than the collapse obtained via one-lung ventilation and induces less lung inflammation. However, iatrogenic pneumothorax can cause paradoxical respiration (collapse during inspiration and expansion during expiration of the nondependent lung) together with an increased mediastinal shift due to loss of negative pressure of non-dependent lung: these alterations may initially decrease the efficiency of ventilation inducing subsequent hypoxemia and hypercapnia, that may be a problem especially during time-consuming procedures without an optimal anaesthetic management. However, temporary hypoxemia is well responsive to $\mathrm{O}_{2}$ supply via facial mask or transnasal high-flow $\mathrm{O}_{2}$ devices. Hypercapnia is generally well tolerated and may be managed using intraoperative manual control or synchronized intermittent mandatory ventilation (SIMV) (13). In addition, "permissive hypercapnia" may improve hemodynamics and reduce ventilation/perfusion mismatch and it's also used during mechanical ventilation (14).

In conclusion, non-intubated anaesthesia during major lung resections should ensure an optimal surgical field allowing a safe lung manipulation in the pleural space, reducing mediastinal movements and cough reflex that can make hazardous hilar and lymph nodes dissection and 
vascular isolation, pivotal steps during an anatomical lung resection.

Dealing with non-intubated major resections, close cooperation between surgical and anaesthesiological teams is mandatory to manage potential complications: uncontrolled bleeding and haemodynamic instability are the main causes of conversion to tracheal intubation. In these cases, rapid intubation in the lateral decubitus performed by a highly trained anaesthesiologist is essential to secure the airways, protect the dependent lung and safely continue the procedure. Other common causes of conversion to tracheal intubation are the presence of dense pleural adhesions, inadequate thoracoscopic vision due to insufficient lung deflation, mediastinal movements due to spontaneous breathing and severe persistent hypoxemia. In these situations too, efficient collaboration between the surgeon and the anaesthesiologist allows completing the surgery as planned.

Concerning emergency management during NITS, Navarro-Martínez et al. proposed an efficient strategy to approach urgent situations applying crisis resource management (CRM) principles. Both clinical and surgical emergencies may occur during NiVATS: in these cases, a quick recognition of the problem and knowing how to deal with it, an established protocol to manage complications and a good teamwork are key points to successfully handle unexpected emergencies (15).

\section{Planning surgery}

Non-intubated VATS lobectomy doesn't technically differ from intubated VATS procedures: either multi and uniportal NiVATS lobectomies are described as feasible and safe.

Iatrogenic pneumothorax obtained through thoracoscopic incisions together with an effective locoregional anaesthesia of the chest wall and vagus nerve allows safe manipulation of the lung and the hilar structures. The quality of lung collapse is comparable to the exclusion obtained during intubated procedures with a double-lumen tube or bronchial blocker (10).

Considering segmentectomy, the identification of the intersegmental planes can be challenging without mechanical ventilation and some alternative techniques have been proposed to identify the cutting intersegmental line: the most common intraoperative techniques are represented by endovascular injection of indocyanine-green (ICG), visible using near-infrared thoracoscopy, and intrabronchial marking with ICG injection under bronchoscopy or electromagnetic navigation bronchoscopy $(16,17)$. In the first case, once the pulmonary artery is clamped or resected, intravenous-injected ICG distributes to the target segment and helps to identify the intersegmental planes. The dividing line is evidenced by a fluorescent boundary between perfused (bright area) and devascularized segments (dark area) (16). In the second case, ICG is instilled in the segmental bronchus, has a longer duration in the alveoli if compared with intravenous ICG administration and no repeated injection is required selective bronchial inflation using a fine needle described by Kamiyoshihara is no longer used due to the risk of air embolism and subsequent severe complications (18).

Once completed lung anatomical resections, air leak test can be performed during NiVATS using AMBU bagassisted ventilation through a facial mask and evaluating the leak during underwater expansion.

Regarding lymph node hilar and mediastinal dissection, it can be efficiently completed during NITS, with results comparable to intubated conventional thoracic surgery: a valid vagal blockade is mandatory to safely perform the procedure avoiding accidental vascular lesions due to broad mediastinal movements and cough reflex.

In the case of segmentectomy for lung cancer, a frozen section of hilar lymph nodes is essential because, in case of a positive result, segmentectomy should be converted to lobectomy to obtain a satisfactory oncological result. However, it should be considered that intentional segmentectomy can be performed in patients who can't functionally tolerate a lobectomy: in these cases, hilar frozen section doesn't change the volume of resection and adjuvant therapy should be evaluated in case of positive N1 or N2 lymph nodes.

Gonzalez-Rivas et al. recently reported a case series of three uniportal NiVATS lobectomies: no complications were reported, the post-operative course was uneventful and all the patients were discharged within the fourth postoperative day (19).

NITS can also be considered a valid alternative for airway surgery: in these cases, the absence of a tracheal tube provides a better surgical field, reducing technical difficulties related to the presence of endotracheal ventilation devices. Jiang et al. reported a retrospective study comparing spontaneous ventilation and intubated VATS tracheal and carinal resections, showing a shorter mean operative time in the first group, with comparable post-operative complications and short-term outcomes (20).

Peng et al. described a successful case of non-intubated VATS carinal reconstruction in a 45 -year-old patient with 
adenoid cystic carcinoma of the lower trachea: after opening the airways, an adequate oxygen supply was provided by high-flow air via the laryngeal mask, obtaining a high oxygen concentration in the pleural cavity. Diaphragmatic and intercostal movements driven by spontaneous respiratory reflexes ensured an adequate oxygen supply and dioxide discharge via main stem bronchi, and no endobronchial tube was needed to support ventilation. The procedure was successfully completed in 230 minutes, without intraoperative complications, proving the feasibility and safety of this technically demanding surgical procedure under spontaneous ventilation, performed by highly trained surgeons in a high-volume NITS centre (21).

Shao et al. reported a case of successful complete thoracoscopic bronchial sleeve resection of right lower lung cancer under non-intubated epidural anaesthesia in a $70 \mathrm{y} / \mathrm{o}$ man, proving that also technically demanding surgical procedures are feasible without tracheal intubation (22).

Considering the surgical management of complications during NITS procedures, Furák et al. described an effective conversion technique from uniportal NiVATS major lung resection to NITS open thoracotomy to manage surgical difficulties: NITS thoracotomy can be performed safely by converting the utility incision to open thoracotomy without using additional medications. Given the excellent results obtained in case of conversion to thoracotomy without intubation, 6 cases of intended open NITS were performed in patients with a low forced expiratory volume in 1 second, with results comparable to intubated surgery (23).

The above reported successful procedures support the technical feasibility and the potential advantages of NiVATS anatomical resections, if performed by skilled thoracic surgeons with a wide VATS experience and an adequate NITS learning curve.

\section{Discussion}

Since the advent of single-lung mechanical ventilation in the late 1960s, general anaesthesia with selective lung exclusion using double-lumen tubes or bronchial blockers has been considered mandatory for major anatomical lung resections, allowing surgical maneuvers on lung, hilar and mediastinal structures. In the last decades, improvements in surgical and anaesthetic techniques have led to a considerable development of minimally invasive thoracic surgery, making it possible to perform also technical demanding major anatomical lung resections via video-assisted thoracoscopic surgery. In recent years, VATS has evolved from multiport to a single incision approach, resulting in a less invasive surgical technique, with less post-operative pain, a reduced hospital stay and a faster recovery.

In this setting of minimally invasive thoracic surgery, non-intubated anaesthesia with spontaneous ventilation is emerging as a feasible and promising technique to reduce intubation-related risks and post-operative adverse effects of general anaesthesia; since the late 1990s, NITS has been applied to minor procedures such as pneumothorax, pleural biopsies and atypical lung resections, with similar surgical results and complication rates compared to intubated surgery (24).

Encouraging results obtained with NITS minor procedures had led to approach also challenging anatomical major lung resections without intubation: the aim of this review is to describe and explore the role of NITS in anatomical major lung resections in terms of feasibility, safety and post-operative outcomes.

Especially in the last decade, a relevant number of studies concerning NITS anatomical resections have been published, showing promising outcomes in terms of technical viability, intra and early post-operative results and complication rates and supporting the feasibility and safety of this innovative approach applied to lung major resections.

In particular, Chen et al. reported the first encouraging results comparing 30 NITS with 30 intubated 3 -port thoracoscopic lobectomies for stage I or II non-small cell lung cancer (NSCLC), describing similar surgical duration, blood loss, and number of dissected lymph nodes between the two groups. Patients who underwent NITS lobectomy had lower rates of sore throat $(6.7 \%$ vs. $40.0 \%, \mathrm{P}=0.002)$ an earlier resumption of oral intake (mean, 4.7 vs. 18.8 hours, $\mathrm{P}<0.001$ ) and shorter postoperative hospital stay (mean, 5.9 vs. 7.1 days, $\mathrm{P}=0.078$ ).

Three patients of the NITS group required conversion to double-lumen tracheal intubation due to persistent hypoxemia, ineffective epidural analgesia and bleeding. One patient in each group required conversion to thoracotomy due to uncontrolled bleeding. These results proved the feasibility and safety of NITS thoracoscopic lobectomy, with comparable surgical results and fewer intubationrelated postoperative complications if compared with traditional VATS lobectomy (25).

Wu et al. analyzed 36 NITS and 48 intubated 3-port thoracoscopic lobectomies for early-stage NSCLC in patients $>65 \mathrm{y} / \mathrm{o}$, reporting comparable operative time, hospital stay, postoperative complication rates and dissected 
Table 1 Main results of the abovementioned comparative studies

\begin{tabular}{|c|c|c|}
\hline Author, journal, year, study design & Patient groups & Main outcomes \\
\hline $\begin{array}{l}\text { AlGhamdi et al., J Thorac Dis } \\
2018 \text { (26), retrospective study }\end{array}$ & $\begin{array}{l}31 \text { NITS and } 31 \text { intubated VATS } \\
\text { lobectomies for early-stage } \\
\text { NSCLC }\end{array}$ & $\begin{array}{l}\text { - Comparable hospital stay, total chest tube duration, anaesthesia } \\
\text { duration, blood loss, postoperative complications, conversion rates } \\
\text { - Fewer dissected lymph nodes in NITS group }\end{array}$ \\
\hline $\begin{array}{l}\text { Xue et al., World J Surg Onc } \\
2021 \text { (28), meta-analysis }\end{array}$ & $\begin{array}{l}8 \text { studies comparing NITS } \\
\text { and intubated major lung } \\
\text { resections (lobectomies and } \\
\text { segmentectomies) }\end{array}$ & $\begin{array}{l}\text { - Comparable surgical duration postoperative complication rates } \\
\text { and number of dissected lymph nodes } \\
\text { - Shorter hospital stay and chest-tube duration in NITS group }\end{array}$ \\
\hline
\end{tabular}

NITS, non-intubated thoracic surgery; NSCLC, non-small cell lung cancer; VATS, video-assisted thoracic surgery.

lymph nodes between the two groups. Only 1 (3\%) NITS lobectomy was converted to tracheal intubation due to prolonged desaturation. This study focuses on the feasibility of NITS anatomical lung resection also in elderly patients and suggests that NITS can be a valid alternative for managing selected geriatric lung cancer patients (6).

A retrospective analysis conducted by AlGhamdi et al. comparing 31 NITS and 31 intubated VATS lobectomies with lymph nodes dissection for early-stage NSCLC reported no differences in postoperative hospitalization period (6.9 vs. 7.6 days, $\mathrm{P}=0.578$ ) and total chest tube duration (5.6 vs. 5.4 days, $\mathrm{P}=0.943$ ), with similar perioperative outcomes in terms of anaesthesia duration, blood loss, postoperative complications and conversion rates. The only reported statistically significant difference was the number of dissected lymph nodes (12.6 in the NITS group $v s .18$ in the intubated group, $\mathrm{P}=0.003$ ) (26).

Moreover, Liu et al. proposed a propensity score matching analysis evaluating the early outcomes of NITS compared to intubated thoracoscopic anatomical lung resections in NSCLC, including 339 patients undergoing VATS anatomical resections (282 lobectomies, of whom 119 NITS procedures, and 57 segmentectomies, of whom 32 NITS procedures). The analysis showed no significant differences between the two groups in terms of operative time, intraoperative blood loss, number of resected lymph nodes and duration of chest tube placement time, with better results in postoperative fasting time and hospital stay in the NITS group, suggesting a more rapid recovery for NITS (27).

Finally, a recent meta-analysis by Xue et al. including 8 studies and evaluating the feasibility and safety of NITS thoracoscopic major resections for NSCLC compared to intubated procedures showed no significant differences in postoperative complication rates, surgical duration and number of dissected lymph nodes, with a shorter hospital stay and chest-tube placement time in patients undergoing NITS (28). Despite the limits of this meta-analysis (few studies included, mainly from Chinese experiences that represent only a part of the global situation, and the use of the random-effect model that could reduce the statistical power due to the heterogeneity of the sample), the reported data confirm that non-intubated surgery can effectively be considered a feasible and safe promising technique to reduce the invasiveness of surgical procedures and shorten the hospital stay (Table 1).

Considering the oncological adequacy of NITS procedures, most of the studies dealing with NITS major 
lung resections reported no significant differences about lymph node dissection; only one of the above reported studies showed a significant difference regarding the number of dissected lymph nodes (27). However, regardless the number of lymph nodes dissected, the required nodal stations were sampled and an adequate oncological resection was maintained also in the NITS group. These results suggest that a satisfactory nodal sampling or dissection is viable also during NITS. Considering that NITS anatomical resections are often proposed for ground glass opacity (GGO) lesions without a solid area, nodal sampling could be adequate to satisfy oncological criteria.

Furthermore, analyzing the oncological value of NITS, Furák et al. retrospectively reviewed 66 NSCLC stage IB-IIIB patients, of which 38 treated with conventional uniportal VATS lobectomy and 28 with NITS VATS uniportal lobectomy, who received adjuvant chemotherapy. The study reported an oncological advantage in NITS patients, showing better compliance with therapy protocols and fewer events of toxicity: these results can be related to a better physiological inflammatory and cellular immune response and fewer immunodepressive effects after a less invasive surgical procedure and a shorter hospital stay. Even though further studies are needed to investigate the relation of NITS and oncological advantages after surgery in NSCLC, these results support the benefits of minimal invasiveness on adjuvant chemotherapy (29).

These conclusions are consistent with the results reported by the randomized controlled trial proposed by Jeon et al. comparing perioperative inflammatory changes in patients after intubated and NITS for NSCLC. Postoperative levels of IL- 6 and TNF- $\alpha$ were significantly lower in NITS group in the early postoperative period, suggesting that NITS procedures may attenuate the early inflammatory cytokine changes after surgery (30).

Only few studies dealing with long-term oncological outcomes after non-intubated lobectomy compared with intubated procedures for early-stage NSCLC are available; a recent propensity-matched analysis including 115 NITS and 155 intubated patients with an average follow-up of 74 months showed no significant differences in recurrence rates (14.4\% vs. $25.8 \%$, respectively; $\mathrm{P}=0.057)$ and overall survival $(7.9 \%$ vs. $93.8 \%$, respectively; $\mathrm{P}=0.144)$ between the two groups (31).

However, despite the technical feasibility and the promising prospects of NITS major lung resections, some recommendations should be taken into account approaching NiVATS, above all a well-established protocol to manage complications. Potential severe complications, represented mainly by uncontrolled bleeding and unstable hemodynamic or respiratory conditions, require an immediate conversion to general anaesthesia to stabilize the patient and efficiently manage the complication. Other common causes of conversion to tracheal intubation are the presence of dense pleural adhesions, inadequate thoracoscopic vision due to insufficient lung deflation, mediastinal movements due to spontaneous breathing and severe persistent hypoxemia. In these cases, an efficient and close cooperation between surgeons and anaesthesiologists is essential to avoid serious consequences and to complete the procedure. A careful selection of patients, an adequate surgical learning curve beginning from minor procedures and a close collaboration between surgeons and anaesthesiologists are pivotal steps to successfully perform NITS major lung resections; knowing the limits of this technique and being able to recognize potential technical difficulties are the key points to avoid critical situations and manage possible complications.

In conclusion, non-intubated major lung resections are an emerging and promising surgical alternative to traditional intubated thoracic surgery: combining minimally invasive surgical and anaesthetic techniques offers advantages in terms of faster recovery and shorter hospital stay, with reduced risks of complications related to tracheal intubation and general anaesthesia.

However, despite these promising results and the technical viability of NITS major lung resections, more studies and a longer follow-up are needed to investigate the feasibility in everyday clinical practice and the oncological value of NITS anatomical lung resections.

\section{Acknowledgments}

Funding: None.

\section{Footnote}

Provenance and Peer Review: This article was commissioned by the Guest Editor (József Furák) for the series "Spontaneous Ventilation Thoracic Surgery" published in AME Surgical fournal. The article has undergone external peer review.

Reporting Checklist: The authors have completed the Narrative Review reporting checklist. Available at https:// asj.amegroups.com/article/view/10.21037/asj-21-79/rc 
Peer Review File: Available at https://asj.amegroups.com/ article/view/10.21037/asj-21-79/prf

Conflicts of Interest: Both authors have completed the ICMJE uniform disclosure form (available at https://asj. amegroups.com/article/view/10.21037/asj-21-79/coif). The series "Spontaneous Ventilation Thoracic Surgery" was commissioned by the editorial office without any funding or sponsorship. DT serves as an unpaid editorial board member of AME Surgical Fournal from January 2021 to December 2022. The authors have no other conflicts of interest to declare.

Ethical Statement: The authors are accountable for all aspects of the work in ensuring that questions related to the accuracy or integrity of any part of the work are appropriately investigated and resolved.

Open Access Statement: This is an Open Access article distributed in accordance with the Creative Commons Attribution-NonCommercial-NoDerivs 4.0 International License (CC BY-NC-ND 4.0), which permits the noncommercial replication and distribution of the article with the strict proviso that no changes or edits are made and the original work is properly cited (including links to both the formal publication through the relevant DOI and the license). See: https://creativecommons.org/licenses/by-nc-nd/4.0/.

\section{References}

1. Yan TD, Black D, Bannon PG, et al. Systematic review and meta-analysis of randomized and nonrandomized trials on safety and efficacy of video-assisted thoracic surgery lobectomy for early-stage non-small-cell lung cancer. J Clin Oncol 2009;27:2553-62.

2. Pompeo E, Mineo D, Rogliani P, et al. Feasibility and results of awake thoracoscopic resection of solitary pulmonary nodules. Ann Thorac Surg 2004;78:1761-8.

3. Al-Abdullatief M, Wahood A, Al-Shirawi N, et al. Awake anaesthesia for major thoracic surgical procedures: an observational study. Eur J Cardiothorac Surg 2007;32:346-50.

4. Buitrago R, Serna A, González-Rivas D, et al. First nonintubated uniportal video-assisted pulmonary lobectomy in America. J Vis Surg 2016;2:144.

5. Diotti C, Mohamed S, Cattaneo M, et al. Non-intubated VATS for lung cancer-a focus on segmentectomy: a narrative review. Video-assist Thorac Surg 2021;6:26.
6. Wu CY, Chen JS, Lin YS, et al. Feasibility and safety of nonintubated thoracoscopic lobectomy for geriatric lung cancer patients. Ann Thorac Surg 2013;95:405-11.

7. Chen KC, Cheng YJ, Hung MH, et al. Nonintubated thoracoscopic surgery using regional anesthesia and vagal block and targeted sedation. J Thorac Dis 2014;6:31-6.

8. Galvez C, Navarro-Martinez J, Bolufer S, et al. Nonintubated uniportal VATS pulmonary anatomical resections. J Vis Surg 2017;3:120.

9. Starke H, Zinne N, Leffler A, et al. Developing a minimally-invasive anaesthesiological approach to nonintubated uniportal video-assisted thoracoscopic surgery in minor and major thoracic surgery. $\mathrm{J}$ Thorac Dis 2020;12:7202-17.

10. Gonzalez-Rivas D, Bonome C, Fieira E, et al. Nonintubated video-assisted thoracoscopic lung resections: the future of thoracic surgery? Eur J Cardiothorac Surg 2016;49:721-31.

11. Piccioni F, Langer M, Fumagalli L, et al. Thoracic paravertebral anaesthesia for awake video-assisted thoracoscopic surgery daily. Anaesthesia 2010;65:1221-4.

12. Hung MH, Chan KC, Liu YJ, et al. Nonintubated thoracoscopic lobectomy for lung cancer using epidural anesthesia and intercostal blockade: a retrospective cohort study of 238 cases. Medicine (Baltimore) 2015;94:e727.

13. Guo Z, Yin W, Pan H, et al. Video-assisted thoracoscopic surgery segmentectomy by non-intubated or intubated anesthesia: a comparative analysis of short-term outcome. J Thorac Dis 2016;8:359-68.

14. Sinclair SE, Kregenow DA, Lamm WJ, et al. Hypercapnic acidosis is protective in an in vivo model of ventilatorinduced lung injury. Am J Respir Crit Care Med 2002;166:403-8.

15. Navarro-Martínez J, Galiana-Ivars M, Rivera-Cogollos MJ, et al. Management of Intraoperative Crisis During Nonintubated Thoracic Surgery. Thorac Surg Clin 2020;30:101-10.

16. Vladimir C, Zdenek K, Lukas F, et al. Clarification of the resection line non-intubated segmentectomy using indocyanine green. Ann Transl Med 2019;7:38.

17. Sekine Y, Ko E, Oishi H, et al. A simple and effective technique for identification of intersegmental planes by infrared thoracoscopy after transbronchial injection of indocyanine green. J Thorac Cardiovasc Surg 2012;143:1330-5.

18. Otsuka T, Nakamura Y, Harada A, et al. Extremely rare but potential complication of diffuse brain edema due to air embolism during lung segmentectomy with selected 
segmental inflation technique by syringe needle during video-assisted thoracoscopic surgery. J Thorac Cardiovasc Surg 2011;142:e151-2.

19. Gonzalez-Rivas D, Yang Y, Guido W, et al. Nonintubated (tubeless) uniportal video-assisted thoracoscopic lobectomy. Ann Cardiothorac Surg 2016;5:151-3.

20. Jiang L, Liu J, Gonzalez-Rivas D, et al. Thoracoscopic surgery for tracheal and carinal resection and reconstruction under spontaneous ventilation. J Thorac Cardiovasc Surg 2018;155:2746-54.

21. Peng G, Cui F, Ang KL, et al. Non-intubated combined with video-assisted thoracoscopic in carinal reconstruction. J Thorac Dis 2016;8:586-93.

22. Shao W, Phan K, Guo X, et al. Non-intubated complete thoracoscopic bronchial sleeve resection for central lung cancer. J Thorac Dis 2014;6:1485-8.

23. Furák J, Szabó Z, Tánczos T, et al. Conversion method to manage surgical difficulties in non-intubated uniportal video-assisted thoracic surgery for major lung resection: simple thoracotomy without intubation. J Thorac Dis 2020;12:2061-9.

24. Mineo TC. Epidural anesthesia in awake thoracic surgery. Eur J Cardiothorac Surg 2007;32:13-9.

25. Chen JS, Cheng YJ, Hung MH, et al. Nonintubated thoracoscopic lobectomy for lung cancer. Ann Surg 2011;254:1038-43.

26. AlGhamdi ZM, Lynhiavu L, Moon YK, et al. Comparison

doi: 10.21037/asj-21-79

Cite this article as: Diotti C, Tosi D. Major lung resections with spontaneous ventilation: a narrative review. AME Surg J 2022;2:17. of non-intubated versus intubated video-assisted thoracoscopic lobectomy for lung cancer. J Thorac Dis 2018;10:4236-43.

27. Liu J, Cui F, Pompeo E, et al. The impact of nonintubated versus intubated anaesthesia on early outcomes of video-assisted thoracoscopic anatomical resection in non-small-cell lung cancer: a propensity score matching analysis. Eur J Cardiothorac Surg 2016;50:920-5.

28. Xue W, Duan G, Zhang X, et al. Comparison of nonintubated and intubated video-assisted thoracoscopic surgeries of major pulmonary resections for lung cancer-a meta-analysis. World J Surg Oncol 2021;19:87.

29. Furák J, Paróczai D, Burián K, et al. Oncological advantage of nonintubated thoracic surgery: Better compliance of adjuvant treatment after lung lobectomy. Thorac Cancer 2020;11:3309-16.

30. Jeon J, Sung S, Moon Y, et al. Comparison of early postoperative cytokine changes in patients undergoing intubated and non-intubated thoracic surgery: a randomized controlled trial. Interact Cardiovasc Thorac Surg 2021;32:343-50.

31. Wang ML, How CH, Hung MH, et al. Longterm outcomes after nonintubated versus intubated thoracoscopic lobectomy for clinical stage I non-small cell lung cancer: A propensity-matched analysis. J Formos Med Assoc 2021;120:1949-56. 